\title{
Glial Cell Line-Derived Neurotrophic Factor (GDNF) for movement disorders. Historical review and current developments
}

\author{
Boeris D Febns ${ }^{1 *}$, Cenzato $\mathrm{M}^{1}$, Dones $\mathrm{F}^{\mathbf{1}}$ and Garbossa $\mathrm{D}^{2}$ \\ ${ }^{1}$ Department of Neurosurgery, Niguarda Hospital, Milan, Italy \\ ${ }^{2}$ Department of Neurosurgery, University of Turin, Italy
}

\begin{abstract}
Convection-enhanced delivery (CED) is a bulk flow-driven process. It allows direct, homogeneous, targeted perfusion of CNS regions with putative therapeutics while bypassing the blood-brain barrier. Delivering therapeutic neurotrophic factors directly to the brain for the treatment of Parkinson's Disease (PD) and other movement disorders has moved researchers in the last decade into this promising field. The glial cell line-derived neurotrophic factor (GDNF) is a well-established trophic agent for dopaminergic (DA) neurons in vitro and in vivo. GDNF is necessary for maintenance of neuronal morphological and neurochemical phenotype and protects DA neurons from toxic damage. Numerous studies on animal models of Parkinson's disease (PD) have reported beneficial effects of GDNF on nigrostriatal DA neuron survival. However, translation of these observations to the clinical setting has been hampered so far by side effects associated with the chronic continuous intra-striatal infusion of recombinant GDNF. We report past and present research investigating the potential of direct drug delivery to the brain for the treatment of PD and other movement disorders.
\end{abstract}

\section{Introduction}

Several preclinical papers based on rodent and non-human primate models had described the benefits of GDNF treatment on nigrostriatal neurons [1]. In early studies, GDNF showed a specific action on survival of rat E16 midbrain DA neurons in culture. It also proved to be a potent and selective stimulator of dopamine uptake and neurite outgrowth in tyrosine hydroxylase positive $(\mathrm{TH}+)$ neurons [2]. These positive in vitro observations led to immediate testing of GDNF effects on PD animal models based on toxin-induced destruction of midbrain DA neurons. Intracranial delivery of GDNF potentiates striatal dopamine system function and midbrain dopaminergic neuronal survival in several models of chemically-induced PD in rodents as well as primates [3].

Although despite initial success in preclinical studies and few early clinical trials, neurotrophic factors have not then shown their ability as neurorestorative therapies in humans. Delivering neurotrophic factors directly to the brain offer the possibility of neurorestoration in neurodegenerative movement disorders such as PD. This work aim is to report on modern and historical drug delivery strategies with a particular focus on the delivery of glial cell-line derived neurotrophic factor (GDNF) for the treatment of PD.

Current milestone therapy for PD is nowadays represented by L-dopa oral therapy. Effective in the early stages of PD, long-term treatment with L-dopa, dopamine agonists, and monoamine oxidase inhibitors is associated with significant Drug Delivery for Movement Disorders motor and psychiatric complications. Motor fluctuations usually manifest as "wearing off" and "on-off" periods. Decline in response to L-dopa is a well-known phenomenon to clinicians over time, as well as "on-off" fluctuations in motor performance that are not clearly related to L-dopa administration. Response to dopamine declines with time and is known to control symptoms but not to address the cause as this pharmacological strategy fails to address the underlying cause of neurodegeneration.

GDNF and the GDNF-family ligands neurturin, artemin, and persephin belong to the transforming growth factor- $\beta$ superfamily, which are a collection of multifunctional cytokines. The neurotrophic effects of GDNF have been found by investigators to be dependent on the presence of transforming growth factor- $\beta$ in both in vitro and in vivo studies.

GDNF and related GDNF-family ligands insist on the Ret tyrosine kinase pathway. Ret mRNA has been demonstrated to be expressed in the substantia nigra of adult rats. This may explain the observed role of GDNF in dopaminergic neuroprotection [4]. GDNF has been proven neurotrophic for noradrenergic, serotoninergic, and cholinergic neurons. These family of neurons are known to be degenerate in PD. PD could potentially have enormous advantages from GDNF infusion as all the current treatments are palliative and are associated with high rates of side effects. This treatment could reverse the degeneration of the dopaminergic pathways almost reversing the pathological process itself.

Few aspects of the molecule itself demonstrated immediately difficult to deal with the high molecular weight of the GDNF homodimer structure prevents transport across the blood-brain barrier. This excluded the systemic option to start with. Overcoming the bloodbrain-barrier (BBB) obstacle for GDNF delivery to the brain using a systemic route has become a major technological challenge

${ }^{\star}$ Correspondence to: Boeris D, Department of Neurosurgery, Niguarda Hospital, Milan, Italy, Tel: 39-392-310-9696; E-mail: boerisdavide@gmail.com

Received: November 20, 2018; Accepted: December 04, 2018; Published: December 10, 2018 
The Trojan horse approach was tested in several ways: 1) systemic administration of nanoliposomes engulfing a GDNF plasmid engineered to cross the BBB [5]. 2) attempt was made to fuse GDNF to a monoclonal immunoglobulin (GDNF-IgG) directed against the BBB cellular component that proved to be potent in mice.

3) biodegradable GDNF-loaded microspheres implanted in the striatum were tested to overcome the BBB problem since they sustainably release recombinant GDNF for 8 weeks [6,7]. More successful appeared to be the administration of GDNF by nasal route, using cationic liposomes to increase their residence time through electrostatic interactions at the olfactory epithelium. Intranasal GDNF given to rats, provided significant protection of striatal DA neurons.

\section{Preclinical studies in rodents}

The effects of GDNF have been assessed in two validated preclinical models of PD which utilize lesions of the nigrostriatal pathway. The most common form of lesioning in rodents is the 6-OHDA model, whilst in non-human primates,1-methyl-4-phenyl-1,2,3,6tetrahydropyridine (MPTP) is utilized to achieve selective depletion of nigrostriatal dopaminergic neurons. Studies of Infusion of recombinant human GDNF and 125 Iodine-labelled GDNF into the ventricular system of non-lesioned rats resulted in the diffusion of GDNF into brain structures including the cortex, septum, diagonal band of Broca, fimbria, striatum, hippocampus, hypothalamus, substantia nigra, ventral tegmental area, and cerebellum GDNF intraventricular infusion resulted also in increased hypothalamic dopamine content, resulting in significant cachexia in experimental animals. Intraventricular infusion of GDNF in 6-OHDA-lesioned rats resulted in locomotor improvements and increased striatal dopamine turnover [8,9]. Reduced weight gain remained a consistent adverse effect. Side effects were considered to be related to the small volume of the rodent brain and proximity of structures to the ventricular system led to studies in nonhuman primates.

\section{Preclinical studies in primates}

Mixed results came out from intraventricular infusion of GDNF in MPTP-lesioned rhesus monkeys and marmosets. Infusion has been associated with Significant improvements in locomotor activity following four monthly infusions at doses ranging from 100-1,000 $\mu \mathrm{g}$ of GDNF, which were correlated with increases in dopamine metabolite concentrations in the substantia nigra, the same was not observed in the putamen [10]. Motor function improvements and reductions in L-dopa-induced dyskinesia have also been observed in marmosets receiving intraventricular GDNF. However, infusions potential was questioned by an autoradiographic study of the distribution of 125 I-GDNF infused into the lateral ventricle of MPTP-lesioned rhesus monkeys, this study clearly demonstrated that in contrast to rodent studies, GDNF did not diffuse effectively into the caudate or putamen [10]. This study questioned whether the success of intraventricular infusions in rodents a consequence of the much smaller diffusion distances in rat brain might be.

\section{Intraventricular infusion of GDNF - First randomized trial}

The first randomized double-blind placebo-controlled trial of intracerebroventricular infusion of GDNF was reported in 2003. The study included 50 patients with moderately advanced L-dopa responsive PD. This study failed to achieve its primary end point. Patients reported substantial side effects without evidence of clinical benefit. Side effects included hyponatremia, anorexia, weight loss, nausea, vomiting, and distressing Lhermitte's phenomenon. Inadequate diffusion of GDNF into nigrostriatal structures was thought to be the key issue in the lack of clinical benefit and overwhelming side effects. This conclusion was subsequently supported by post-mortem analysis in one of the trial subjects that did not demonstrate evidence of dopaminergic neuronal recovery.

\section{Intracerebral infusion of GDNF in rodents}

Injection of GDNF into the substantia nigra or striatum after of 6-OHDA lesioning has been shown to be neuroprotective, and neurorestorative [11-14]. Intrastriatal delivery of GDNF demonstrated superior to intranigral administration by protecting the entire nigrostriatal pathway. When delivering GDNF into the substantia nigra this does not protect striatal axons from degeneration and does not prevent locomotor disability but is very effective in preventing cell death within the substantia nigra itself [15]. GDNF when delivered into the striatum at the time of striatal lesioning, however, does preserve motor function suggesting that the effect of GDNF on dopaminergic neurons differs according to whether it is applied to axons or cell bodies [16]. GDNF can induce functional improvements following severe 6-OHDA lesioning of the striatum causing neurochemical changes in the striatum, the globus pallidus interna and externa, and the substantia nigra [17].

\section{Intraputamenal infusion of GDNF in non-human primates}

The effects of continuous intraputaminal delivery of GDNF has been reported in 2002 in both normal aged and MPTP-lesioned nonhuman primates [17-18]. GDNF infused directly into the putamen has been reported to increase dopaminergic cell size and increased cells number within the substantia nigra. Fiber density improvements throughout the striatum and globus pallidus has also been reported. GDNF increases dopamine and its metabolites in the striatum and globus pallidus as well, according to the authors. This 2002 study concluded that intraputamenal infusions of GDNF in MPTP lesioned primates and intact aged monkeys was associated with improvements in the primate PD rating scale, improved general motor performance, and increased hand speed [18]. Unfortunately, in 2007, the promising results of non-human primate studies were countered by the results of a 6-month chronic infusion toxicity study in rhesus monkeys. This study raised a number of safety concerns over continuous unilateral intraputamenal infusions of recombinant human GDNF at a flow rate of $150 \mu \mathrm{L} /$ day. Four cut off doses were used in the study $0,15,30$, or 100 $\mu \mathrm{g} /$ day. In the fourth group, both behavioural and pathological markers of toxicity were observed including reduced food intake and weight loss, meningeal thickening, and multifocal cerebellar Purkinje cell loss. The observation supported an association with chronic GDNF infusion at high doses and cerebellar toxicity. However, subsequent analysis indicated that Purkinje cell loss may have been a consequence of abrupt GDNF withdrawal.

\section{Intraputamen infusion of GDNF in humans}

Two different clinical studies have investigated the effect of GDNF administered directly to the posterodorsal putamen, which is known to be the site of greatest dopaminergic neuronal depletion in PD. The first study reported in 2005 and was an open-label study of continuous intraputamenal delivery of GDNF via stereotactically placed microcatheters attached to a subcutaneous infusion pump placed in the anterior abdominal wall. In this first human study five subjects with poorly controlled symptoms by medical treatment were enrolled. Five out of five subjects demonstrated improvement in both clinically 
and neuroradiologically using 18 F-dopa PET imaging parameters. Neuroimages using 18 F-dopa PET confirmed increased dopamine storage capacity in both the putamen and SN [12].

As a result of this promising study subjects were entered into a 12-month extension study, which resulted in sustained improvement. Post-mortem analysis was possible in one subject who died of causes unrelated to the study revealed a marked increase in tyrosine hydroxylase-positive nerve fibers, and possibly neuronal sprouting in the SN on the side of intraputamenal GDNF administration when compared to the contralateral (un-infused) side [19-22].

A second open-label study enrolled ten subjects with different catheters, infusion parameters, and dosing regimens compared to the first study [23]. All ten subjects showed reductions in UPDRS, improvements in postural stability, dyskinesia, end-of-dose fluctuations and quality of life scores. The promising results of these 2 openlabel studies led to the commencement of a multicentre randomized controlled trial.

\section{Multicentre randomized trial of intraputamenal GDNF infusion}

Thirty-four subjects were randomized to receive either bilateral intraputamenal infusions of $15 \mu \mathrm{g} /$ putamen/day GDNF or placebo [24]. At 6 months, subjects receiving GDNF had failed to demonstrate the predetermined level of clinical improvement in the primary endpoint (reduction in UPDRS motor score in the "off" state) required to achieve statistical significance despite improvements in 18F-dopa PET parameters. A further concern was the detection of neutralizing antibodies in 3 patients, and an additional 3 patients developed serious device-related adverse effects. The results of this randomized study, taken in conjunction with the 6-month toxicity study in primates which demonstrated cerebellar Purkinje cell loss [21], led to the withdrawal of GDNF and cessation of clinical trials. Also, an in-depth analysis of why the randomized trial failed despite promising results in the initial openlabel studies followed. A lack of standardization of catheter design and surgical technique across centers is likely to have contributed to the failure of the phase II trial. Salvatore et al. [25] analyzed the distribution of $125 \mathrm{I}$-GDNF in the putamen of rhesus monkeys when delivered using the same delivery system as in the phase II study. This analysis revealed significant variability in drug distribution, with the majority of GDNF restricted to the location of the catheter tip. This study concluded that if translated to the human putamen, the bioavailability of GDNF would have been limited to only $2-9 \%$ of the total putamen volume and that point source concentration of GDNF could explain the failure to achieve the expected clinical improvement.

\section{Convection enhanced delivery infusion}

The role of suboptimal drug delivery in the failure of the phase II trial has led to significant research interest in convection-enhanced delivery (CED), and in particular optimization of drug distribution in the putamen. CED describes the direct delivery of drugs to the brain through intraparenchymal microcatheters. By employing high infusion flow rates and establishing a pressure gradient at the tip of the catheter, CED confers several potential advantages over traditional drug injection techniques, including homogeneous distribution throughout large and clinically relevant volumes of brain tissue and reduced tissue trauma. In the 20 years since its inception, it has become clear that a number of important factors govern successful CED - catheter design and diameter, method of implantation, accuracy of targeting, infusion regime employed, architecture of the target tissue in the brain, and the physic-chemical characteristics of the infused agent.
Image-guided CED of GDNF, using real-time MRI tracking of distribution within the putamen, has been reported in rhesus monkeys [26] and resulted in the effective distribution of GDNF throughout the putamen, but was associated with leakage of GDNF into perivascular spaces. For this reason, as well as the potentially toxic effects associated with chronic infusion in non-human primates, intermittent delivery of GDNF has been proposed as a more "physiological" strategy. Rationale for Chronic Intermittent CED, the requirement for intermittent CED is of particular relevance to the delivery of GDNF where chronic infusion has been associated with poor distribution and local toxicity due to point source accumulation [24, 25,27]. Prolonged intracerebral drug infusions have been achieved in recent clinical trials with subcutaneously implanted pumps and access ports [26-30]. However, a number of complications have been reported which are attributable to the use and re-filling of subcutaneous devices as well as infection rates of up to $12 \%$ [29]. Infectious complications may be a consequence of entraining bacteria into the subcutaneous plane around the implanted device during needle insertion. The dermis is also known to have a high density of immune cells, and stimulation of an immune response due to subcutaneous spillage during needle insertion into the pump reservoir may explain the detection of anti-GDNF antibodies (including neutralizing antibodies) in 3 trial subjects in Lang et al. [24] phase II trial of intraputamenal infusion of GDNF. Subcutaneous pumps pose substantial challenges for CED trials. Multiple catheters require the implantation of multiple pumps as no reliable distributor is currently available, which allows a single pump to deliver infusate concurrently through more than one catheter, which places an additional burden on patients. To effectively use implantable pumps for prolonged drug delivery, extensive analysis of drug stability at body temperature is required to mitigate the risk of accumulation of degraded drug products within the pump reservoir and to ensure that there is no loss of biological activity of the infused drug. An implantable transcutaneous port has the potential to prevent the complications associated with subcutaneous access devices and pumps by allowing thorough cleansing of the port prior to needle insertion, by eliminating the need to penetrate skin to replenish the therapeutic agent, and by facilitating intermittent connection to an external pump device. Bone-anchored transcutaneous devices have proven more effective than skin-anchored devices in longterm clinical studies, which is likely to be a result of greater stability at the skin/device interface [30]. The natural world has provided a highly successful model for transcutaneous bone-anchored medical devices in the form of deer's antlers [31]. These imperatives have led to the development of a transcutaneous bone anchored port and implantable multi-catheter system for chronic intermittent CED [32,33]. This novel implantable catheter system with transcutaneous bone-anchored port is currently being used in a phase II double-blinded randomized controlled trial of chronic intermittent CED of GDNF for PD in Bristol, UK. In this trial, two microcatheters are implanted bilaterally into each putamen using a robot-assisted and image verified technique. By optimizing the drug distribution through clinically relevant volumes of the putamen and employing an intermittent infusion regime, it is hoped that the failures of GDNF administration of the past can be addressed leading to a neurorestorative treatment for PD. This study has recently published nine months results and did not meet the primary end-point but an extension study is in progress.

\section{Conclusions}

This review demonstrates the interest that the scientific community has for this research field. In vitro results have built enormous expectations for this therapeutic option for Parkinson disease, but so far 
none of the available studies have clearly demonstrated the efficacy and tolerability of this drug delivered onto brain cells. Weather the delivery system or the drug itself is to be redesigned is not clear yet. In the years to come thanks to the recent research the pathway will become clearer.

\section{References}

1. Lonser RR, Sarntinoranont M, Morrison PF, Oldfield EH (2015) Convection-enhanced delivery to the central nervous system. J Neurosurg 122: 697-706. [Crossref]

2. Lin LF, Doherty DH, Lile JD, Bektesh S, Collins F (1993) GDNF: a glial cell linederived neurotrophic factor for midbrain dopaminergic neurons. Science 260: 11301132. [Crossref]

3. Ibáñez CF, Andressoo JO (2017) Biology of GDNF and its receptors - Relevance for disorders of the central nervous system. Neurobiol Dis 97: 80-89. [Crossref]

4. Allen SJ, Watson JJ, Shoemark DK, Barua NU, Patel NK (2013) GDNF, NGF and BDNF as therapeutic options for neurodegeneration. Pharmacol Ther 138: 155-175. [Crossref]

5. Boado RJ, Pardridge WM (2009) Comparison of Blood-Brain Barrier Transport of Glial-Derived Neurotrophic Factor (GDNF) and an IgG-GDNF Fusion Protein in the Rhesus Monkey. Drug Metab Dispos 37: 2299-2304. [Crossref]

6. Fu A, Zhou QH, Hui EK, Lu JZ, Boado RJ, et al. (2010) Intravenous treatment of experimental Parkinson's disease in the mouse with an IgG-GDNF fusion protein that penetrates the blood-brain barrier. Brain Res 1352: 208-213. [Crossref]

7. Aubert-Pouëssel A, Venier-Julienne MC, Clavreul A, Sergent M, Jollivet C, et al. (2004) In vitro study of GDNF release from biodegradable PLGA microspheres. $J$ Control Release 95: 463-475. [Crossref]

8. Migliore MM, Ortiz R, Dye S, Campbell RB, Amiji MM, et al. (2014) Neurotrophic and neuroprotective efficacy of intranasal GDNF in a rat model of Parkinson's disease. Neuroscience 274: 11-23. [Crossref]

9. Lapchak PA, Miller PJ, Collins F, Jiao S (1997) Glial cell line-derived neurotrophic factor attenuates behavioural deficits and regulates nigrostriatal dopaminergic and peptidergic markers in 6-hydroxydopamine-lesioned adult rats: comparison of intraventricular and intranigral delivery. Neuroscience 78: 61-72. [Crossref]

10. Lapchak PA, Araujo DM, Hilt DC, Jiao S, Collin F, et al. (1998) Topographical distribution of [125I]-glial cell line-derived neurotrophic factor in unlesioned and MPTP-lesioned rhesus monkey brain following a bolus intraventricular injection. Brain Res 789: 9-22. [Crossref]

11. Iravani MM, Costa S, Jackson MJ, Tel BC, Cannizzaro C, et al. (2001) GDNF reverses priming for dyskinesia in MPTP-treated, L-DOPA-primed common marmosets. Eur J Neurosci 13: 597-608. [Crossref]

12. Costa S, Iravani MM, Pearce RK, Jenner P (2001) Glial cell line-derived neurotrophic factor concentration dependently improves disability and motor activity in MPTPtreated common marmosets. Eur J Pharmacol 412: 45-50. [Crossref]

13. Nutt JG, Burchiel KJ, Comella CL, Jankovic J, Lang AE, et al. (2003) Randomized, double-blind trial of glial cell line-derived neurotrophic factor (GDNF) in PD. Neurology 60: 69-73. [Crossref]

14. Sauer H, Rosenblad C, Björklund A (1995) Glial cell line-derived neurotrophic factor but not transforming growth factor beta 3 prevents delayed degeneration of nigral dopaminergic neurons following striatal 6-hydroxydopamine lesion. Proc Natl Acad Sci U S A 92: 8935-8939. [Crossref]

15. Kearns CM, Cass WA, Smoot K, Kryscio R, Gash DM (1997) GDNF protection against 6-OHDA: time dependence and requirement for protein synthesis. J Neurosci 17: 71117118. [Crossref]

16. Winkler C, Sauer H, Lee CS, Björklund A (1996) Short-term GDNF treatment provides long-term rescue of lesioned nigral dopaminergic neurons in a rat model of Parkinson's disease. J Neurosci 16: 7206-7215. [Crossref]
17. Kirik D, Rosenblad C, Bjorklund A (2000) Preservation of a functional nigrostriatal dopamine pathway by GDNF in the intrastriatal 6-OHDA lesion model depends on the site of administration of the trophic factor. Eur J Neurosci 12: 3871-3882. [Crossref]

18. Lapchak PA, Miller PJ, Jiao S (1997) Glial cell line-derived neurotrophic factor induces the dopaminergic and cholinergic phenotype and increases locomotor activity in aged Fischer 344 rats. Neuroscience 77: 745-752. [Crossref]

19. Grondin R, Zhang Z, Yi A, Cass WA, Maswood N, et al. (2002) Chronic, controlled GDNF infusion promotes structural and functional recovery in advanced parkinsonian monkeys. Brain 125: 2191-2201. [Crossref]

20. Hovland DN Jr, Boyd RB, Butt MT, Engelhardt JA, Moxness MS, et al. (2007) Sixmonth continuous intraputamenal infusion toxicity study of recombinant methionyl human glial cell line-derived neurotrophic factor (r-metHuGDNF in rhesus monkeys. Toxicol Pathol 35: 1013-1029. [Crossref]

21. Patel NK, Bunnage M, Plaha P, Svendsen CN, Heywood P, et al. (2005) Intraputamena infusion of glial cell line-derived neurotrophic factor in PD: a two-year outcome study. Ann Neurol 57: 298-302. [Crossref]

22. Love S, Plaha P, Patel NK, Hotton GR, Brooks DJ, et al. (2005) Glial cell line-derived neurotrophic factor induces neuronal sprouting in human brain. Nat Med 11: 703-704. [Crossref]

23. Slevin JT, Gash DM, Smith CD, Gerhardt GA, Kryscio R, et al. (2006) Unilateral intraputaminal glial cell line-derived neurotrophic factor in patients with Parkinson disease: response to 1 year each of treatment and withdrawal. Neurosurg Focus 20 E1. [Crossref]

24. Lang AE, Gill S, Patel NK, Lozano A, Nutt JG, et al. (2006) Randomized controlled trial of intraputamenal glial cell line-derived neurotrophic factor infusion in Parkinson disease. Ann Neurol 59: 459-466. [Crossref]

25. Salvatore MF, Ai Y, Fischer B, Zhang AM, Grondin RC, et al. (2006) Point source concentration of GDNF may explain failure of phase II clinical trial. Exp Neurol 202 497-505. [Crossref]

26. Gimenez F, Krauze MT, Valles F, Hadaczek P, Bringas J, et al. (2011) Image-guided convection-enhanced delivery of GDNF protein into monkey putamen. Neuroimage 54: S189-195. [Crossref]

27. Morrison PF, Lonser RR, Oldfield EH (2007) Convective delivery of glial cell linederived neurotrophic factor in the human putamen. J Neurosurg 107: 74-83. [Crossref]

28. Sonabend AM, Stuart RM, Yun J, Yanagihara T, Mohajed H, et al, (2011) Prolonged intracerebral convection-enhanced delivery of topotecan with a subcutaneously implantable infusion pump. Neuro Oncol 13: 886-893. [Crossref]

29. Bogdahn U, Hau P, Stockhammer G, Venkataramana NK, Mahapatra AK, et al. (2011) Targeted therapy for high-grade glioma with the TGF-beta2 inhibitor trabedersen: results of a randomized and controlled phase IIb study. Neuro Oncol 13: 132-142. [Crossref]

30. Niranjan A, Lunsford LD, Richardson RM (eds): Current Concepts in Movemen Disorder Management. Prog Neurol Surg. Basel, Karger, 2018, vol 33, pp 243-252.

31. Pendegrass CJ, Goodship AE, Blunn GW (2006) Development of a soft tissue seal around bone-anchored transcutaneous amputation prostheses. Biomaterials 27: 41834191. [Crossref]

32. Pendegrass CJ, Goodship AE, Price JS, Blunn GW (2006) Nature's answer to breaching the skin barrier: an innovative development for amputees. J Anat 209: 59-67. [Crossref]

33. Barua NU, Hopkins K, Woolley M, O'Sullivan S, Harrison R, et al. (2016) A nove implantable catheter system with transcutaneous port for intermittent convectionenhanced delivery of carboplatin for recurrent glioblastoma. Drug Deliv 23: 167-173. [Crossref]

34. Barua NU, Woolley M, Bienemann AS, Johnson DE, Lewis O, et al. (2013) Intermittent convection-enhanced delivery to the brain through a novel transcutaneous boneanchored port. J Neurosci Methods 214: 223-232. [Crossref]

35. Intraputamenal Convection-Enhanced Delivery. [abstract]. Mov Disord 2017; 32.

Copyright: $\bigcirc 2018$ Febns BD. This is an open-access article distributed under the terms of the Creative Commons Attribution License, which permits unrestricted use, distribution, and reproduction in any medium, provided the original author and source are credited. 